\title{
VOLUMETRIC EVALUATION OF UPPER LIMB FOR THE BREAST CANCER ASSOCIATED LYMPHEDEMA
}

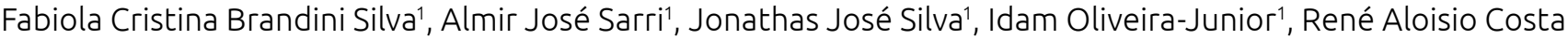
Vieira ${ }^{1}$

${ }^{1}$ Hospital de Câncer de Barretos, Barretos - (SP), Brazil

Introduction: Lymphedema is one of the main complications associated with the treatment of breast cancer. Multiple methodologies can be used in the diagnosis of lymphedema. A simple and clean, easy methodology is lacking, aiming to correlate with the findings of volumetry. Objectives: To evaluate different methodologies for the evaluation of indirect volumetry, by calculating the Frustrum and potential cutoff points related to direct volumetry. Methods: Study approved by the Ethics Committee under number 782/2014. A cross-sectional study with prospective collection, diagnostic evaluation, performed in patients with breast cancer undergoing surgical treatment of the breast. The participants were subjected to evaluations of the upper limbs using volumetry (total limb and hand), cylindrical and radial perimetry for diagnosis of lymphedema at equidistant points every $5 \mathrm{~cm}$ (V10) and $10 \mathrm{~cm}$ (V6). One software was created based on the cylindrical and diametrical measurements of the upper limbs. Concomitant upper limb and hand volumetry was performed, with a difference of $200 \mathrm{~mL}$ in volume being considered a lymphedema. The formula VFC $=\mathrm{h}(\mathrm{C} 12+\mathrm{C} 1 \mathrm{C} 2+\mathrm{C} 22) / 12 \pi$ was used for the cylindrical Frustrum (VFC), and for the elliptical Frustrum (VFE) the formula VFE= (L $\pi / 3) A 2 B-a 2 b) /(A-a)$ was used. To compare the agreement of the volumes, the Interclass Correlation Index was used. In order to equalize values associated with volumetry, the ROC curve was used. Grant from FAPESP project no. 2014 08197-0 and Institutional IEP/HCB no 5/2017. Results: 300 patients were evaluated. With regard to VFE, the result was not adequate in $25.5 \%$ in the V10 assessment and in $8.9 \%$ in the V6. The correlation between the difference in volume in total volume and the difference in volume without hands was excellent (ICC $=0.993$ ). The difference of the risk member was compared with the contralateral of the VC10 and VC6 volumes with hand and VC10 and VC6 without the hand, the intra-class correlation coefficients were 0.840 , $0.878,0.835,0.878$, respectively. The presence or absence of the hand did not change the correlation, given the difference in volume (dV). Considering the difference in volume of the limbs of $200 \mathrm{~mL}$, in the VFC formula dVC6 this value corresponded to $108.79 \mathrm{~mL}$, with a sensitivity of 88.9, specificity of 87.3 and accuracy of 87.7; and in the VFC dVC10 formula this value was $96.9 \mathrm{~mL}$, with a sensitivity of 92.1, specificity of 85.7 and accuracy of 87 . Conclusions: The use of cylindrical Frustrum proved to be satisfactory, and different cutoff points should be used in clinical practice. The creation of the software facilitates the evaluation because it is a clean and reproducible methodology. 